\title{
OPTIMAL SUPERIMPOSED PILOT SELECTION FOR OFDM CHANNEL ESTIMATION
}

\author{
Angiras R. Varma, Chandra R. N. Athaudage \\ ARC Special Research Center for \\ Ultra-Broadband Information Networks \\ University of Melbourne, Australia
}

\author{
Lachlan L. H. Andrew \\ Department of Computer Science \\ California Institute of Technology \\ California, USA
}

\author{
Jonathan H. Manton \\ Research School of Information \\ Sciences and Engineering \\ The Australian National University \\ Canberra. Australia
}

\begin{abstract}
This paper presents an optimal strategy for utilizing superimposed pilots for OFDM channel estimation using Wiener filtering. An algorithm is formulated to determine the optimal rectangular set of time-frequency samples for channel estimation for a given complexity. The proposed scheme shows an improved performance at high Doppler frequencies. Moreover, the separable implementation of the $2 \mathrm{D}$ Wiener filter leads to a significant reduction in complexity with negligible degradation in channel estimation performance. The sensitivity of the proposed technique to channel statistical mismatches is also analyzed. Numerical results demonstrate the superior performance of the proposed technique compared to conventional selection of square set of time-frequency samples for Wiener-filter based channel estimation.
\end{abstract}

\section{INTRODUCTION}

Orthogonal frequency division multiplexing (OFDM) is a spectrally efficient modulation scheme for high-bit-rate wireless communication over multipath fading channels. It is expected that OFDM will play a major role in next generation (B3G and 4G) mobile wireless systems [1]. For coherent modulation schemes estimation of the wideband OFDM channel consisting of large number of subcarriers is important for receiver equalization and data detection. Specifically when the mobility of the receivers is high (high Doppler channels) channel estimation becomes a challenging task.

The conventional channel estimation techniques for OFDM use known symbols or pilots. In these techniques pilots and information symbols are multiplexed in time and/or frequency. As an alternative technique, arithmetically adding pilot symbols to information symbols (superimposing the pilot symbols to the information symbols) has recently attracted wide attention [2][3][4]. Though this technique of superimposed pilots was first proposed for single-carrier systems [2], it has also been used for channel estimation in multicarrier systems such as OFDM systems [4][5]. The main advantage of superimposed pilot scheme is that the information symbols can be transmitted over all time-frequency slots, hence saving the bandwidth compared to time-multiplexed pilot scheme. In addition to this, in the OFDM context, none of the subcarriers need to be dedicated completely or partially for the pilots. In rapidly varying channels (in time or in frequency) superimposed pilots have an advantage in terms of improved channel tracking performance [6]. In [4], the potential of the superimposed pilot scheme for high data rate transmission has been demonstrated.

One of the channel estimation techniques that has been proposed for OFDM systems is two dimensional (time/frequency)
Wiener filtering [7]. Wiener filters have been studied for channel estimation and interpolation with time-multiplexed pilot scheme in OFDM systems [8][9]. Minimum mean square estimation (MMSE) channel estimation technique using Wiener filtering and superimposed pilot training was proposed in [4][5]. However in [4], after the Fast Fourier Transform (FFT) operation, the time-frequency samples are taken for channel estimation from a region chosen arbitrarily, without considering the fading statistics. For instance [4] chooses a square region on the time-frequency plane.

In contrast, this paper proposes an optimum rectangular region for time-frequency sample selection along with its performance. To reduce the complexity of two dimensional Wiener filtering, we propose combining separable Wiener filters [8] with superimposed training. Since the performance of optimal time-frequency sample selection scheme as well as channel estimation scheme depends on the channel's fading statistics (Doppler frequency and delay spread), an analysis is presented to study the sensitivity of the proposed schemes to statistical mismatches.

\section{SyStem MODEL}

\section{A. Channel Model}

The complex baseband model of a wireless time-varying finite impulse response channel can be given as

$$
z(t, \tau)=\sum_{i=0}^{L-1} A_{i}(t) \delta\left(\tau-\tau_{i}\right)
$$

where $A_{i}(t)$ is the time-varying amplitude of the $i$ th path, $\tau_{i}$ is the delay of the $i$ th path and $L$ is number of propagation paths. The amplitude of each path is assumed to be a Rayleigh fading process and the power delay profile of the channel is taken as exponentially decaying. The Fourier transform of $z(t, \tau)$ with respect to the delay $\tau$ is $h_{c}(t, f)$. Under these conditions the joint time-frequency correlation function of $h_{c}(t, f)$ becomes [8]

$$
\begin{aligned}
\Phi(\Delta t ; \Delta f) & =\Phi_{t}(\Delta t) \Phi_{f}(\Delta f) \\
\Phi_{t}(\Delta t) & =J_{0}\left(2 \pi f_{d} \Delta t\right) \\
\Phi_{f}(\Delta f) & =\frac{1-e^{\left(-T_{\mathrm{cp}}\left(1 / \tau_{\mathrm{rms}}+j 2 \pi \Delta f\right)\right)}}{\left(1-e^{\left(-T_{\mathrm{cp}} / \tau_{\mathrm{rms}}\right)}\right)\left(1+j 2 \pi \Delta f \tau_{\mathrm{rms}}\right)}
\end{aligned}
$$

where $\Delta f$ and $\Delta t$ are the separation in frequency and time over which the correlation is measured. The Doppler freuqncy and the cyclic prefix (CP) length of the OFDM symbols are given by $f_{d}$ and $T_{\mathrm{cp}}$, respectively. Root mean square 
(RMS) value of the delay spread is given by $\tau_{\text {rms }}$. OFDM symbol length without cyclic prefix is defined as $T_{s}$ and the total symbol interval including cyclic prefix is defined as $T=T_{s}+T_{c p}$. Inter subcarrier frequency spacing is defined as $F_{s} . N$ is the number of subcarriers and $N_{g}$ is the number of samples in cyclic prefix.

\section{B. Signal Model}

Consider an OFDM system with superimposed pilots, where pilots symbols are arithmetically added to the information symbols at all time-frequency indices before IFFT-operation at the transmitter. The received symbol $y(m, n)$ after FFT operation at a time-frequency grid point $(m, n)$ can be given as

$$
y(m, n)=h(m, n)[s(m, n)+c(m, n)]+w(m, n)
$$

where $h(m, n)=h_{c}\left(m T, n F_{s}\right), s(m, n)$ is the information symbol, $c(m, n)$ is the superimposed pilot and $w(m, n)$ is the noise sample. The time and frequency index can be in the range $0 \leq m \leq M-1$ and $0 \leq n \leq N-1$. Channel estimation in this context is studied using Wiener filtering in the next section.

\section{Channel Estimation}

\section{A. Two Dimensional Wiener Filter}

Optimal channel estimate in the Linear MMSE (LMMSE) sense can be obtained using a two dimensional Wiener filter. The least squares (LS) estimate of the channel is obtained in the first step and these estimates are filtered using a two dimensional Wiener filter to obtain a better channel estimate. Without losing generality we consider estimation of the channel, $h(m, n)$, at an arbitrary time-frequency index $(m, n)$, where $0 \leq m \leq M-1,0 \leq n \leq N-1$. Here $N$ represents the number of subcarriers in the OFDM system, whereas $M$ can take a value till infinity if an infinite time transmission is assumed. The LS estimate of the channel, $\tilde{h}(m, n)$, is obtained by dividing the received symbol after the FFT operation, $y(m, n)$ expressed in (5), by the superimposed pilot $c(m, n)$ as [5]

$$
\widetilde{h}(m, n)=h(m, n)+h(m, n) s^{\prime}(m, n)+w^{\prime}(m, n)
$$

where $s^{\prime}(m, n)=s(m, n) / c(m, n)$ and $w^{\prime}(m, n)=$ $w(m, n) / c(m, n)$. The second and third terms in (6) show the noise introduced by the information symbols and channel noise. To filter out this noise a two dimensional Wiener filter is applied and the channel estimate is $\hat{h}(m, n)=\mathbf{w}_{2 D}^{*}(m, n) \tilde{\mathbf{h}}$, where $\tilde{\mathbf{h}}$ is a $M N \times 1$ vector containing all the elements $\tilde{h}(m, n), 0 \leq m \leq M-1,0 \leq n \leq N-1$ and $\mathbf{w}_{2 D}(m, n)$ is

$$
\mathbf{w}_{2 D}(m, n)=\left[\mathbf{C}_{\mathbf{h h}}+\left(\sigma_{s^{\prime}}^{2}+\sigma_{w^{\prime}}^{2}\right) \mathbf{I}\right]^{-1} \mathbf{C}_{\mathbf{h} h(m, n)}
$$

where $\mathbf{C}_{\mathbf{h h}}$ is the channel autocorrelation function of dimension $M N \times M N$ and $\mathbf{C}_{\mathbf{h} h(m, n)}$ is the cross-covariance vector of dimension $M N \times 1$ obtained from (2)[5]. The symbol $\sigma^{2}$ represents the variance of the corresponding variable.

To reduce the complexity and to alleviate edge effect to a great extend, we consider a subset of $\{y(m, n) \mid 0 \leq m \leq$ $M-1,0 \leq n \leq N-1\}$ for channel estimation. Given a subset of the complete set of time-frequency samples, we can precompute the time-frequency invariant weighting vector. For a given complexity (or for a given number of time-frequency samples in the subset), how can we choose the optimal subset of samples that minimizes the channel estimation error? This question is addressed in the next section.

\section{B. Optimal Selection of Time-Frequency Samples}

Considering the estimation of channel at $(m, n)$, according to MMSE theory, estimation error is minimized by selecting the neighborhood consisting of points with maximum correlation to the point $(m, n)$. We can see that the most correlated sample points to $\tilde{h}(m, n)$ would minimize the MMSE of channel estimation. Thus the optimal region that encompasses the highly correlated samples is a contour of channel timefrequency correlation function surface $\Phi(\Delta t, \Delta f)$. The algorithm to select $C_{\max }$ highly correlated samples is presented as follows:

- Compute $\Phi=\{\Phi(i, j)\}$ for all combinations of $-N \leq$ $i \leq N,-M \leq j \leq M$

- Select set of indices $\mathbf{I}=\{(i, j)\}$ corresponding to $C_{\max }$ number of largest values in $\Phi$.

This method of selection of sample points is used as the benchmark for performance in Section IV.

We propose to use an optimal rectangular region to approximate the optimal region considering following reasons. (i) The performance in terms of MMSE channel estimation (presented in Section IV) using a rectangular region is very close to that of optimal region at low as well as high Doppler frequencies. (ii) It is possible to compute a low complexity separable Wiener filter as discussed in Section III-C. Thus the objective is to choose a rectangular region encompassing the most highly correlated time-frequency samples such that the number of samples within the rectangle satisfies the complexity requirement. The complexity requirement specifies the finite number of time-frequency samples that can be used for channel estimation.

We initially analyze the problem of determining the optimal rectangular time-frequency region for channel estimation in continuous domain and then convert to discrete domain for further analysis. Without losing generality a rectangular region encompassing the time-frequency sample $(m, n)$ is calculated as follows. Let the height of the rectangle be $2 f_{0}$ in frequency direction and the width be $2 t_{0}$ in time direction. The total accumulated correlation in this rectangle is

$$
\Phi_{C}\left(t_{0}, f_{0}\right)=\int_{-f_{0}}^{f_{0}} \int_{-t_{0}}^{t_{0}}|\Phi(\Delta t, \Delta f)| d \Delta t d \Delta f
$$

where $\Phi(\Delta t, \Delta f)$ is time-frequency channel correlation function as given in (2). Since time and frequency correlation functions are separable $\Phi(\Delta t, \Delta f)=\Phi_{t}(\Delta t) \Phi_{f}(\Delta f)$, the complexity constrain, and using the inter-carrier and inter-time slot spacing, gives

$$
\frac{4 f_{0} t_{0}}{F_{s} T} \leq C_{\max }
$$


Let $F_{s} T C_{\max }=K$. Applying (9) and separability of $\Phi(\Delta t, \Delta f),(8)$ can be modified as

$$
\Phi_{C}\left(f_{0}\right)=\int_{-f_{0}}^{f_{0}}\left|\Phi_{f}(\Delta f)\right| d \Delta f \int_{-K / f_{0}}^{K / f_{0}}\left|\Phi_{t}(\Delta t)\right| d \Delta t .
$$

The objective is to find $f_{0}$ that maximizes $\Phi_{C}\left(f_{0}\right)$. The time correlation function $\Phi_{t}(\Delta t)=J_{0}\left(2 \pi f_{d} \Delta t\right)$ can be approximated as a polynomial function, which is given as [10]

$J_{0}\left(2 \pi f_{d} \Delta t\right) \simeq \sum_{m=0}^{n} c_{n, m}\left(2 \pi f_{d} \Delta t\right)^{2 m}, 0 \leq 2 \pi f_{d} \Delta t \leq 2 n$,

where $c_{n, m}$ is given by $c_{n, m}=\left((-1)^{m} n^{1-2 m}(n+m-\right.$ $1) !) /\left(2^{2 m}(n-m) !(m !)^{2}\right)$. Assuming $T_{\mathrm{cp}}$ to be large compared to the channel delay spread $\tau_{\text {rms }}$, the frequency correlation function can be approximated as $\Phi_{f}(\Delta f)=1 /(1+$ $\left.j 2 \pi \Delta f \tau_{\text {rms }}\right)$. Using the above expressions for $\Phi_{t}(\Delta t)$ and $\Phi_{f}(\Delta f)$, total correlation function $\Phi_{C}\left(f_{0}\right)$ is obtained as

$$
\begin{array}{r}
\Phi_{C}\left(f_{0}\right)=\frac{2}{b} \sum_{m=0}^{n} \frac{c_{n, m}\left(2 \pi f_{d}\right)^{2 m} K^{2 m+1}}{(2 m+1) f_{0}^{2 m+1}} . \\
\ln \left|b f_{0}+\sqrt{b^{2} f_{0}^{2}+1}\right|,
\end{array}
$$

where $b=2 \pi \tau_{\text {rms }}$. For $n=1$, the approximation (12) becomes

$$
\Phi_{C}\left(f_{0}\right)=\left[\frac{2 K}{\pi \tau_{\mathrm{rms}} f_{0}}-\frac{2 \pi f_{d}^{2} K^{3}}{3 \tau_{\mathrm{rms}} f_{0}^{3}}\right] \ln \left|b f_{0}+\sqrt{b^{2} f_{0}^{2}+1}\right| .
$$

For maximization of (13) numerically, it is beneficial to convert $f_{0}$ from continuous domain to discrete domain as $f_{0}=\eta F_{s}, 1 \leq \eta \leq N$, where $F_{s}$ is subcarrier spacing and $\eta$ is subcarrier index. The terms in (13) $\tau_{\mathrm{rms}} f_{0}$ can be modified as $\tau_{\mathrm{rms}} f_{0}=\tau_{\mathrm{rms}} \eta F_{s}=\tau_{\mathrm{rms}} \eta / T_{s}=\tilde{\tau}_{\mathrm{rms}} \eta / N$, where $\tilde{\tau}_{\text {rms }}$ is the RMS delay spread normalized by sampling period, $T_{s} / N$. Similarly the term $f_{d} / f_{0}$ in (13) can be modified as $f_{d} / f_{0}=f_{d} / \eta F_{s}=f_{d} T_{s} / \eta=\tilde{f}_{d} / \eta \epsilon$, where $\tilde{f}_{d}=f_{d} T$ is normalized Doppler frequency and $\epsilon=\left(1+N_{g} / N\right)$. The discrete expression for total correlation function $\Phi_{C}\left(\eta F_{s}\right)=$ $\Phi_{C}^{\prime}(\eta)$ is

$$
\Phi_{C}^{\prime}(\eta)=\left(\frac{A}{\eta}-\frac{B}{\eta^{3}}\right) \ln \left|c \eta+\sqrt{c^{2} \eta^{2}+1}\right|,
$$

where $A=2 K N / \pi \tilde{\tau}_{\mathrm{rms}}, B=2 \pi K^{3} \tilde{f}_{d}^{2} N / 3 \tilde{\tau}_{\mathrm{rms}} \epsilon^{2}$ and $c=$ $2 \pi \tilde{\tau}_{\text {rms }} / N$. The steps to be executed to obtain the optimal rectangle is as follows:

1) Compute $\eta_{\max }=\arg \max _{\eta} \Phi_{C}^{\prime}(\eta), \eta=1,2, \ldots N$

2) Compute $M^{\prime}=\left\lfloor C_{\max } / \eta_{\max }+0.5\right\rfloor$, where $M^{\prime}$ is the discrete dimension of the rectangle in terms of number of OFDM symbols in time direction and $\lfloor x\rfloor$ denotes the largest integer not exceeding $x$.

3) Calculate $N^{\prime}=\left\lfloor C_{\max } / M^{\prime}\right\rfloor$, where $N^{\prime}$ is discrete dimension of the rectangle in terms of number of subcarriers.

The performance of optimal time-frequency sample selection is demonstrated in Section IV.

\section{Separable Wiener Filter}

The complexity of the two dimensional Wiener filter can be significantly reduced by incorporating two one dimensional Wiener filters with minor sacrifice in the channel estimation performance. The idea is to obtain channel estimates in frequency direction first, utilizing frequency correlation function and then smooth the channel estimates using a second Wiener filter in time direction utilizing time correlation function [8]. Here we are utilizing the separability of the time-frequency channel correlation function given in (2). Considering stationarity of the channel, both the filters are time and frequency invariant. The separable Wiener filter algorithm has the following two steps:

\section{Step 1}

Construct a vector of LS channel estimates from samples in frequency direction, centered at time index $k$ and frequency index $n$.

$$
\tilde{\mathbf{h}}(k, n)=\left[\tilde{h}\left(k, n+n^{\prime}\right) \mid-N_{1} \leq n^{\prime} \leq N_{2}\right]
$$

where $N_{1}=N_{2}=\left\lfloor N^{\prime} / 2\right\rfloor$ if $N^{\prime}$ is odd and $N_{1}=N^{\prime} / 2$ and $N_{2}=N^{\prime} / 2-1$ if $N^{\prime}$ is even. $N^{\prime}$ is the height of the rectangle in frequency direction obtained from the optimization procedure in Section III-B. Obtain the channel estimate $\hat{h}_{1}(k, n)$ using Wiener filter as

$$
\hat{h}_{1}(k, n)=\mathbf{w}_{1}^{*} \tilde{\mathbf{h}}(k, n)
$$

where $\mathrm{w}_{1}$ is of the form

$$
\mathbf{w}_{1}=\left[\mathbf{C}_{\mathbf{h}(k, n) \mathbf{h}(k, n)}^{f}+\sigma^{2} I\right]^{-1} \mathbf{C}_{\mathbf{h}(k, n) h(k, n)}^{f}
$$

and

$$
\mathbf{h}(k, n)=\left[h\left(k, n+n^{\prime}\right) \mid-N_{1} \leq n^{\prime} \leq N_{2}\right]
$$

The correlation functions $\mathbf{C}_{\mathbf{h}(k, n) \mathbf{h}(k, n)}^{f}$ and $\mathbf{C}_{\mathbf{h}(k, n) h(k, n)}^{f}$ are independent of the specific channel realization, and can be precomputed from the channel frequency correlation function in (4) and $\sigma^{2}=\sigma_{s^{\prime}}^{2}+\sigma_{w^{\prime}}^{2}$.

\section{Step 2}

Construct a vector of channel estimates from (16) in time direction centered around the time index $m$ and frequency index $n$

$$
\hat{\mathbf{h}}_{1}(m, n)=\left[\hat{h}\left(m+m^{\prime}, n\right) \mid-M_{1} \leq m^{\prime} \leq M_{2}\right]
$$

where $M_{1}=M_{2}=\left\lfloor M^{\prime} / 2\right\rfloor$ if $M^{\prime}$ is odd and $M_{1}=M^{\prime} / 2$ and $M_{2}=M^{\prime} / 2-1$ if $M^{\prime}$ is even. $M^{\prime}$ is the length of the optimal rectangle in time direction obtained in Section III-B. Estimate $\hat{h}(m, n)$ from $\hat{\mathbf{h}}_{1}(m, n)$ using a Wiener smoothing filter

$$
\hat{h}(m, n)=\mathbf{w}_{2}^{*} \hat{\mathbf{h}}_{1}(m, n)
$$

where $\mathbf{w}_{2}^{*}=\mathbf{C}_{h(m, n) \hat{\mathbf{h}}_{1}(m, n)} \cdot \mathbf{C}_{\hat{\mathbf{h}}_{1}(m, n) \hat{\mathbf{h}}_{1}(m, n)}^{-1}$. The $(i, j)$ th element of the auto-correlation matrix $\mathbf{C}_{\hat{\mathbf{h}}_{1}(m, n) \hat{\mathbf{h}}_{1}(m, n)}$ is given by (21). $\mathbf{C}_{\mathbf{h}(i, n) \mathbf{h}(j, n)}^{t}(i, j)$ is the $(i, j)$ th element of the time correlation function obtained using (3) and $\mathbf{C}_{\mathbf{h h}}^{f}$ of 


$$
\mathbf{C}_{\hat{\mathbf{h}}_{1}(m, n) \hat{\mathbf{h}}_{1}(m, n)}(i, j)=\mathbf{w}_{1}^{*}\left[\mathbf{C}_{\mathbf{h}(i, n) \mathbf{h}(j, n)}^{t}(i, j) \mathbf{C}_{\mathbf{h h}}^{f}+\sigma^{2} \mathbf{I}(i, j)\right] \mathbf{w}_{1} .
$$

dimension $N^{\prime} \times N^{\prime}$ is obtained using the frequency correlation function (4). The $i$ th element of cross-covariance vector $\mathbf{C}_{h(m, n) \hat{\mathbf{h}}_{1}(m, n)}$ is obtained as

$$
\mathbf{C}_{h(m, n) \hat{\mathbf{h}}_{1(m, n)}}(i)=\mathbf{C}_{h(m, n) h(i, n)}^{t} \mathbf{C}_{h(m, n) \mathbf{h}(i, n)}^{f} \mathbf{W}_{1}
$$

where $\mathbf{C}_{h(m, n) h(i, n)}^{t}$ and $\mathbf{C}_{h(m, n) \mathbf{h}(i, n)}^{f}$ are computed from (3) and (4). In (21), $\sigma^{2}(i, j)=\sigma_{s^{\prime}}^{2}+\sigma_{w^{\prime}}^{2}$ when $i=j$ otherwise $\sigma^{2}(i, j)=\sigma_{s^{\prime}}^{2}$. The channel estimation error (MMSE) $\mathcal{E}$ for the separable Wiener filter case can be given as

$$
\mathcal{E}=\mathbf{C}_{h h}-\mathbf{C}_{h \hat{\mathbf{h}}_{1}} \mathbf{C}_{\hat{\mathbf{h}}_{1} \hat{\mathbf{h}}_{1}}^{-1} \mathbf{C}_{\hat{\mathbf{h}}_{1} h}
$$

where $h=h(m, n)$ and $\hat{\mathbf{h}}_{1}=\hat{\mathbf{h}}_{1}(m, n)$. The individual terms in (23) are obtained using expressions given in Step 2. The above MMSE expression is used for performance evaluation in Section IV.

\section{Complexity Reduction With Separable Wiener Filter}

Assuming that the channel correlation functions in time and frequency are available, the Wiener filter coefficients can be computed offline. In a separable filter, calculations to estimate the channel at a particular $(m, n)$ can be reused to estimate channel at its neighboring points. This reduces the number of multiplications required to estimate a single channel gain for the separable Wiener filter to $M^{\prime}+N^{\prime}$. For the 2D Wiener filter the number of multiplications per channel gain estimation is $M^{\prime} N^{\prime}$. Thus, using two one dimensional Wiener filters provides a complexity reduction factor of $\left(M^{\prime}+N^{\prime}\right) / M^{\prime} N^{\prime}$. Numerical performance comparison of separable Wiener filter with two dimensional Wiener filter is presented in Section IV.

\section{E. Effect of Errors in Channel Statistics}

Optimal sample selection in Section III-B and channel estimation techniques presented in Section III-C depends on the statistics of the channel (Doppler frequency and RMS delay-spread). Two possible situations can be analyzed in this context. First we assume that there is estimators to estimate Doppler frequency and RMS delay-spread at the receiver, hence estimation error is assumed to be at low range. Second, receiver is working at a fixed $\tilde{f}_{d}$ and $\tilde{\tau}_{\text {rms }}$, which are decided at design time. Hence these statistical parameters can be far away from reality. In both these cases it is important to see how the channel estimation performance is affected by the statistical errors. The channel estimation error (MMSE) for this case is given by

$$
\mathcal{E}^{\prime}=\mathbf{C}_{h h}-\mathbf{C}_{h \mathbf{h}} \mathbf{w}-\mathbf{w}^{*} \mathbf{C}_{\mathbf{h} h}-\mathbf{w}^{*} \mathbf{C}_{\mathbf{h h}} \mathbf{w}
$$

where $\mathbf{w}$ is the channel estimator constructed with inaccurate channel statistics. Results on channel estimation error in presence and absence of statistical error are given in Section IV-C.

\section{Numerical Results}

This section provides numerical results based on analytical expressions derived in previous sections, demonstrating the benefits of the proposed optimal pilot selection for channel estimation. We consider an OFDM system with $N=64$ subcarriers and cyclic prefix length is 6 samples. The channel is assumed to be Rayleigh fading with exponentially decreasing power delay profile. The channel has normalized RMS delay spread $\left(\tilde{\tau}_{\text {rms }}\right)$ of 0.5 . Normalized Doppler frequency, $\tilde{f}_{d}$, is considered in the range $0.001-0.4$. The normalized Doppler frequency is chosen in this range to model high mobility in future mobile networks. Average channel SNR is assumed to be $20 \mathrm{~dB}$ and the optimal power allocation for superimposed pilot for this channel SNR is $20 \%$ of the total power [11].

\section{A. Performance of Optimal Time-Frequency Sample Selection}

Performance of optimal time-frequency sample selection is compared in Fig 1. This compares the mean square channel estimation error versus normalized Doppler frequency while selecting pilots from an optimal region, optimal rectangular region and a square region. The number of samples selected for channel estimation is up to a maximum of $C_{\max }=100$. As given in Fig. 1, at low Doppler frequency performance of all the three schemes are similar. However at high Doppler frequencies the performance of rectangular window and optimal window becomes better than the square window. The

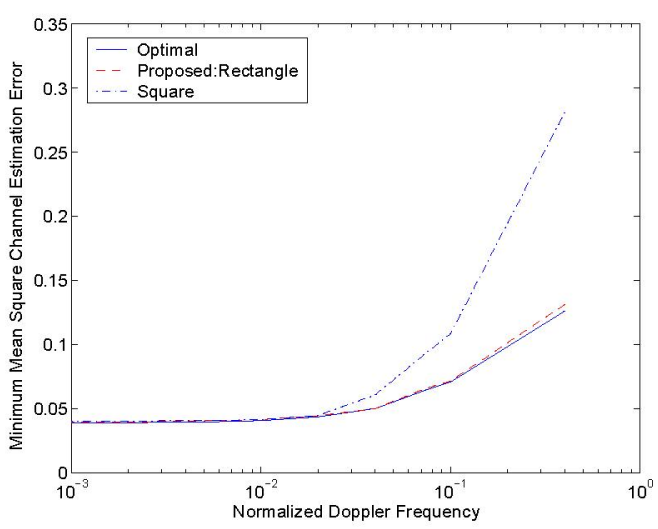

Fig. 1. Performance comparison of optimal sample selection with rectangular and square approximations.

dimensions of the optimal rectangular regions are given in Table I.

\section{B. Comparison of $2 D$ Filter and Separable Filter}

In this section the performance of the two dimensional Wiener filter is compared with the separable Wiener filter. For the same time-frequency sample region channel estimation error performance of 2D Wiener filter is compared with the performance of separable Wiener filter. The number of samples used for channel estimation is up to a maximum 
TABLE I

PROPOSED DIMENSIONS OF RECTANGULAR REGIONS FOR DIFFERENT NORMALIZED DOPPLER FREQUENCIES.

\begin{tabular}{|c|c|c|c|c|c|c|c|c|}
\hline$\tilde{f}_{d}$ & 0.001 & 0.005 & 0.007 & 0.01 & 0.02 & 0.04 & 0.1 & 0.4 \\
\hline$N^{\prime}$ & 3 & 5 & 7 & 9 & 10 & 20 & 33 & 50 \\
\hline$M^{\prime}$ & 33 & 20 & 14 & 11 & 10 & 5 & 3 & 2 \\
\hline
\end{tabular}

of $C_{\max }=100$. For both the schemes height and width of the rectangle encompassing the time-frequency samples were optimized using the method proposed in Section IIIB. Fig 2 plots the minimum mean square channel estimation error against normalized Doppler frequency. The performance graph shows that both schemes perform similarly when the number of samples used for channel estimation is the same. However the complexity of the separable Wiener filter is just $\left(M^{\prime}+N^{\prime}\right) / M^{\prime} N^{\prime}$ times that of the 2D Wiener filter.

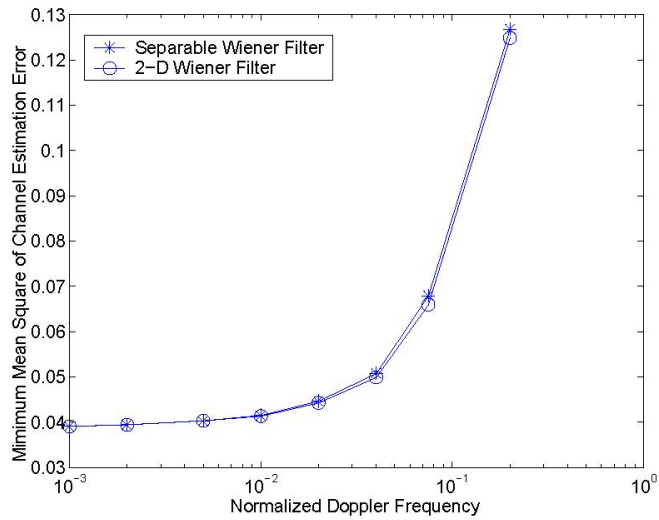

Fig. 2. Performance of two dimensional Wiener filter versus separable Wiener filter.

\section{Performance in Presence of Statistical Mismatch}

Statistical mismatch occurs when the receiver uses a wrong estimate of the Doppler frequency. We assume that the RMS delay-spread is obtained with no error. The over estimate of the Doppler frequency is assumed to be known with percentage errors $20 \%$ and $150 \%$, where $20 \%$ is considered as a worst case bound on the error in estimating Doppler frequency. The large $150 \%$ error is considered for the case where an assumption is made on the Doppler frequency at the receiver design stage which can be far out from the actual value. Both these scenarios are possible in reality. The number of time-frequency samples used for channel estimation is up to a maximum of $C_{\max }=100$. Fig 3 shows channel estimation error vs Doppler frequency for different errors in obtaining Doppler frequency. The optimal rectangular region for selecting time-frequency samples shows lesser sensitivity to statistical mismatch, whereas the scheme using the square region for sample selection shows much higher sensitivity (larger performance degradation) to statistical mismatch.

\section{CONCLUSION}

In the context of superimposed pilots, the proposed rectangular approximation to the optimal time-frequency sample

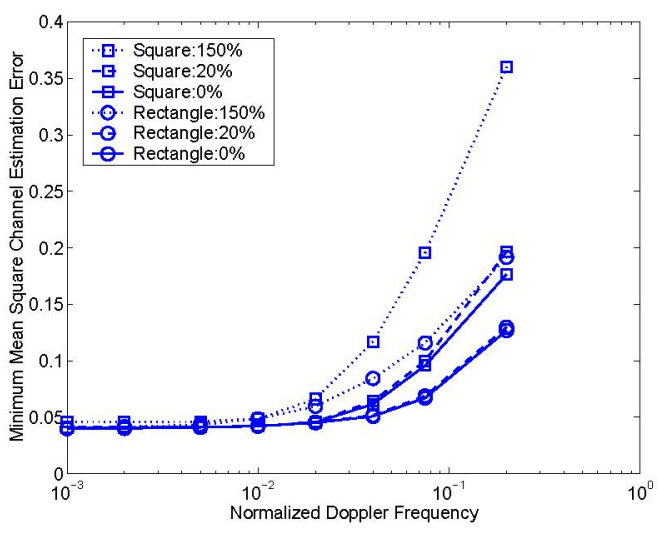

Fig. 3. Performance comparison of optimal rectangular sample selection with square approximation under Doppler frequency mismatches.

selection is reasonable with only small reduction in performance. Rectangular approximation leads to the separable implementation of the Wiener filter, thus reducing the complexity significantly. A square region of samples is not an effective choice at high Doppler frequencies as the channel estimation performance is reduced compared to the optimal selection as well as the rectangular approximation. The rectangular approximation of sample selection region has the additional benefit being less sensitive to channel statistical mismatch.

\section{ACKNOWLEDGMENT}

This work was supported by the Australian Research Council.

\section{REFERENCES}

[1] S. Hara and R. Prasad, Multicarrier Techniques for $4 G$ Mobile Communications, Artech House, first edition, 2003.

[2] B. Farhang-Boroujeny, "Pilot-based channel identification: proposal for semi-blind identification of communication channels," Electronics Letters, vol. 31, no. 13, pp. 1044 - 1046, June 1995.

[3] J. H. Manton, I. M. Y. Mareels, and Y. Hua, "Affine precoders for reliable communications," in Proc. IEEE ICASSP, Istanbul, Turkey, June 2000 , vol. V, pp. 2749-2752.

[4] S. Balasubramanian, B. Farhang-Boroujeny, and V.J. Mathews, "Pilot embedding for channel estimation and tracking in OFDM systems," in Proceedings of IEEE Globcom, Nov. 2004, vol. 2, pp. $1244-1248$.

[5] C.K. Ho, B. Farhang-Boroujeny, and F. Chin, "Added pilot semi-blind channel estimation scheme for OFDM in fading channels," in PrOC IEEE Globcom, Nov. 2001, vol. 3075 - 3079, pp. 3075 - 3079 .

[6] M. Dong, L. Tong, and B. M. Sadler, "Optimal insertion of pilot symbols for transmissions over time-varying flat fading channels," IEEE Transactions on Signal Processing, vol. 52, no. 5, pp. 1403 - 1418, 2004.

[7] P. Hoeher, S. Kaiser, and P. Robertson, "Two-dimensional pilot-symbolaided channel estimation by wiener filtering," in Proceedings of IEEE ICASSP, Apr. 1997, vol. 3, pp. 1845 - 1848.

[8] M. Sandell and O. Edfors, "A comparitive study of pilot-based channel estimators for wireless OFDM," Research Report TULEA, vol. 31, SEP 1996, [online] http:/epubl.luth.se/avslutade/0347-0881/9619/sae96r.pdf.

[9] C. R. N. Athaudage and A. D. S. Jayalath, "Enhanced MMSE channel estimation using timing error statistics for wireless ofdm systems," IEEE Trans Broadcast, vol. 50, no. 4, pp. 369-376, 2004.

[10] R. P. Millane and J. L. Eads, "Polynomial approximations to Bessel functions," IEEE Transactions on Antennas and Propagation, vol. 51, no. 6 , pp. $1398-1400,2003$.

[11] D. H. Pham and J. H. Manton, "Orthogonal superimposed training on linear precoding: a new affine precoder design," in Proc. SPAWC 2005, June 2005, pp. 455-449. 